\title{
Effect of Levitation Forces on the Performance of Surface Micromachined MEMS Gyroscopes
}

\author{
Chris Painter \\ Microsystems Laboratory, University of California, Irvine \\ Irvine, CA, USA \\ cpainter@uci.edu
}

\begin{abstract}
In this paper, we study the effect of electrostatic levitation forces on the performance of a MEMS surface micromachined gyroscope. An error model relating the effects of the induced levitation deflections to scale factor and cross axis sensitivity is presented. Simulation and experimental results on a surface micromachined test structure are used to identify deflection versus voltage characteristics. These characteristics are scaled based off typical gyroscope parameters and the findings are that levitation forces can cause more than $50 \%$ reduction in scale factor and more than .1\% increase in undesirable cross-axis sensitivity.
\end{abstract}

\section{Keywords}

Gyroscope, Levitation, Scale Factor, Cross Axis Sensitivity

\section{INTRODUCTION}

Levitation force is a phenomenon occurring in electrostatically actuated MEMS devices where a structure meant to move parallel to the substrate is forced away from the substrate due to electrical fringing fields [1] (Figure 1). In surface micromachined devices, where thickness of structures is on the order of $2 \mu \mathrm{m}$, the out-of-plane stiffness is typically on the same order as the in-plane stiffness, which can result in substantial vertical deflections. Surface micromachined gyroscopes that measure rotations about the axis perpendicular to the substrate are designed to vibrate parallel to the substrate and any orthogonal motion is nondesirable and effects performance of the device.

To determine the impact of this effect, it is necessary to develop models relating levitation force to gyro performance and to determine the relationship between design parameters and levitation forces. Toward this goal, we develop an analytical model which captures the various performance altering effects of levitation forces, including scale factor variation and cross axis sensitivity. It has been shown in prior work [2] that it is difficult to distinguish levitation forces from the effect of electronic parasitics using electronic sensing, and thus difficult to qualitatively characterize the effect on gyro performance. Because levitation force is dependent on both geometry and in-plane position of the device, we have developed deflection models using a levitation tester where in-plane motion is suppressed, decoupling geometric and position influence on the electrostatic force. To decouple

\author{
Andrei Shkel \\ Microsystems Laboratory, University of California, Irvine \\ Irvine, CA, USA \\ ashkel@uci.edu
}

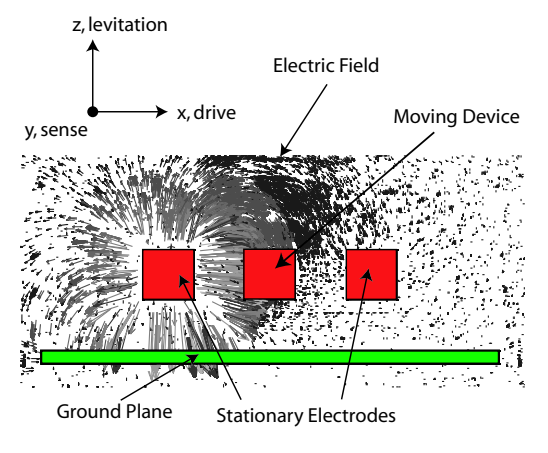

Figure 1. Levitation in surface micromachined gyroscopes is due to non-symmetrical fringing fields in the presence of a ground plane in close proximity to the device and drive/sense electrodes.

motional effects from electronic sensing parasitics, computer simulation and experiments based on optical detection are performed.

\section{GYROSCOPE ERROR MODEL}

A typical implementation of a $\mathrm{z}$-axis Coriolis vibratory rate gyroscope can be approximated as a single mass-springdamper system which uses control circuitry to maintain a constant amplitude of oscillation along a drive direction $x$, whereby rotation is measured by the Coriolis force induced vibration along an orthogonal axis $y$. While the ideal motion of the device during operation is in a two dimensional plane, levitation forces induced by the drive voltages result in motion orthogonal to this plane along a $z$ direction. The levitation force is nonlinear and will be comprised of a plurality of frequency harmonics. However, filtering is typically done in gyroscopes to eliminate all out of band and out of phase noise and as such, we need only to consider the effect of levitation force components that exist at the drive frequency of the device. The equations of motion under the assumption of constant amplitude in the drive and some level of levitation induced motion $z(t)$ is

$$
\begin{aligned}
x(t) & =X_{D} \sin \left(\omega_{n} t\right) \\
\ddot{y}+\frac{\omega_{n}}{Q} \dot{y}+\omega_{n}^{2} y & =-2 \Omega_{z} \dot{x}+2 \Omega_{x} \dot{z} \\
z(t) & =z_{b}+Z_{D} \sin \left(\omega_{n} t\right)
\end{aligned}
$$

where $\omega_{n}^{2}=k / m$ is the natural frequency squared, $c / m=$ 


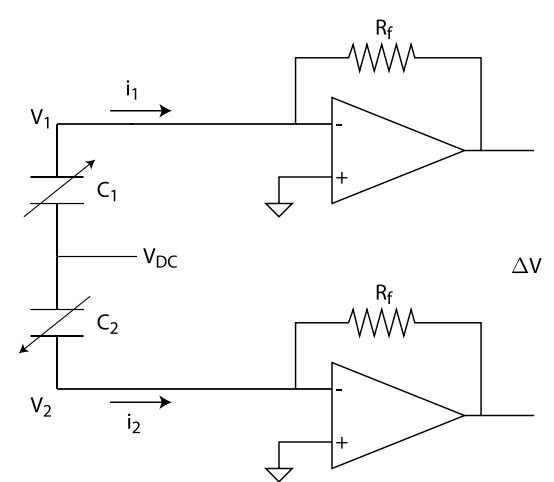

(a)

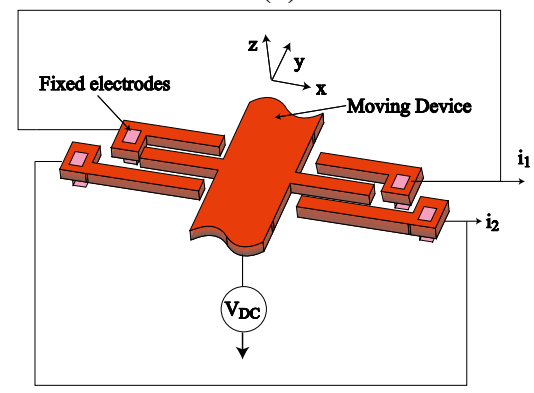

(b)

Figure 2. (a) The gyroscope sense electronics convert motional current into an output voltage based off (b) change in capacitance of the oscillating structure.

$\omega_{n} / Q$ is the damping to mass ratio, $\Omega$ is a constant input angular velocity, $X_{D}$ is the oscillation amplitude in the drive direction, $z_{b}$ is static levitation due to DC bias applied between the mass and electrodes, and $Z_{D}$ is the oscillatory $z$ amplitude deflection component in band with the drive frequency. Based on the proposed mathematical model, the sense amplitude is solved to be

$$
|y(t)|=\frac{2 Q}{\omega_{n}}\left(\Omega_{z} X_{D}-\Omega_{x} Z_{D}\right)
$$

The response from the gyroscope in the sense direction induces a motional current which is converted to a voltage and differentially measured (Figure 2a). The magnitude is proportional to the capacitance values (Figure 2b), calculated from instantaneous height $z_{0}-z$, comb overlap $x_{0}$, and comb separation $y_{0} \pm y$

$$
\begin{aligned}
& C_{1}=\frac{\epsilon_{0} x_{0}\left(z_{0}-z\right)}{y_{0}-y} \\
& C_{2}=\frac{\epsilon_{0} x_{0}\left(z_{0}-z\right)}{y_{0}+y}
\end{aligned}
$$

The output voltage is derived as

$$
\Delta V=\left(\frac{\partial\left(C_{1}-C_{2}\right)}{\partial y} \dot{y}+\frac{\partial\left(C_{1}-C_{2}\right)}{\partial z} \dot{z}\right) R_{f} V_{D C}
$$

In gyroscopes, the sense deflection is typically small compared to the gap $\left(y \ll y_{0}\right)$. Under this small deflection

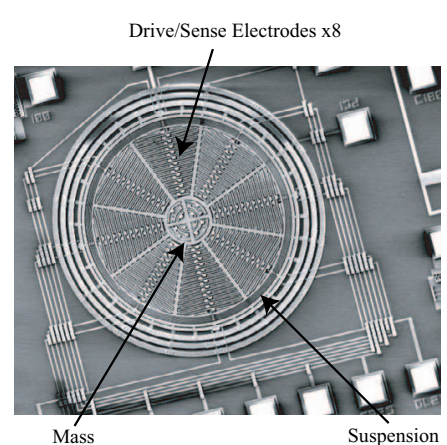

(a)

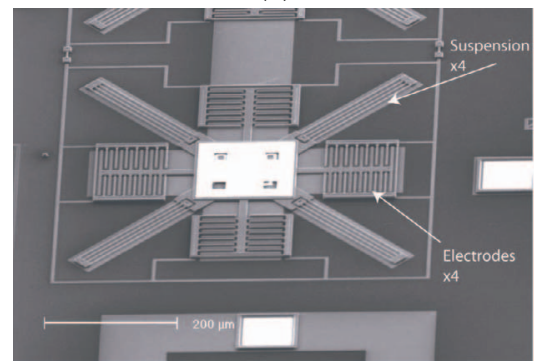

(b)

Figure 3. (a) The effect of levitation on a gyroscope is approximated through experiments done on a (b) surface micromachined levitation tester which is compliant only in the out-of-plane direction

assumption, the output voltage is

$$
|\Delta V|=\frac{4 \epsilon_{0} x_{0}\left(z_{0}-z\right)}{y_{0}^{2}} Q R_{f} V_{D C}\left(\Omega_{z} X_{D}-\Omega_{x} Z_{D}\right)
$$

where the $\left(z_{0}-z\right)$ term influences scale factor of the gyroscope and $\Omega_{x} Z_{D}$ represents the cross axis sensitivity. Since the static deflection is typically much larger than the vertical oscillation, $z$ in the scale factor term is assumed approximately equal to $z_{b}$. Thus, for a typical gyroscope implementation, we would expect a large variation in scale factor compared to change in cross-axis sensitivity.

\section{LEVITATION TESTER}

To decouple the influence of in-plane motion on levitation forces, we fabricated a test structure (Figure 3b) which mimics the electrode structure of a typical gyroscope [3] (Figure $3 \mathrm{a}$ ), but has a set of four folded suspension members which suppress in-plane $x$ and $y$ motion while allowing out-of-plane $z$ motion. The device was fabricated using the MEMSCAP MUMPS surface micromachining process. The suspension and mobile combs are made from polysilicon (POLY1 mask layer) with a thickness of two microns. A conductive ground plane made from .5 micron thick polysilicon (POLY0 mask layer) is spaced two microns from the bottom of the POLY1 layer. The suspension beams have length of 200 microns and width of two microns. The fixed drive electrodes are 3 microns wide, the mobile electrodes are 4 microns wide, and 


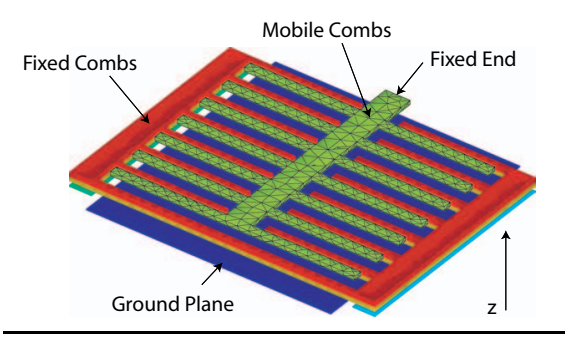

Figure 4. Multi-physics simulation of the levitation tester. Because of similarity conditions, one quadrant can be modeled, reducing analysis time.

the spacing between the electrodes is 4 microns. Each mobile comb has a $48 \mu \mathrm{m}$ engagement length with one fixed comb on either side of the mobile comb. There are 14 engagements per quadrant and 4 quadrants on the device.

\section{FINITE ELEMENT MODELING}

Simulations to characterize the levitation forces are performed using the Coventor multi-domain finite element software package. A reduced order model consisting of a single quadrant of the device is used to lower computation time (Figure 4). In the quadrant model, a voltage is applied between the mobile and fixed structure and the electrostatic force is calculated at $.1 \mu \mathrm{m}$ vertical translation steps of the mobile combs. This process is repeated for voltage values of 50,70 , and $100 \mathrm{~V}$. The reaction forces are scaled by a factor of four, to account for the contribution of all four quadrants, and plotted as a function of vertical translation. The translation is multiplied by the spring constant for the suspension and also plotted as a function of vertical translation. The folded beam suspension is a well known type of suspension with a spring constant $k$ of

$$
k=\frac{4 E w t^{3}}{l^{3}}
$$

The equilibrium position for a voltage is determined by the intersection of the suspension force and the electrostatic force.

\section{EXPERIMENTS}

\section{Optical Profilerometer}

To verify the simulation results, experiments using optical detection were performed. The first experiment uses the PL $\mu$ non-contact profilerometer developed by Sensofar [4] (Figure 5a). The levitation tester is first completely scanned to verify parallelism of the mirror with the substrate during actuation. Any tilt of the entire chip is compensated using algorithms included in the software package. The cross section of one of the beams is scanned (Figure 5b) and the distance between the top of the Poly0 ground and the top of the Poly 1 beam is measured. The device is actuated using a DC power supply for voltages of $0,50,70$, and $100 \mathrm{~V}$.

\section{Vibrometer}

The second experiment is performed using a non-contact laser Doppler vibrometer developed by Polytec PI [5] by scanning a single point on top of the device (Figure 5c). As the vibrometer was designed for frequency response, it was necessary to actuate the devices with a low frequncy $(.1 \mathrm{~Hz})$ sawtooth signal using maximum voltages of 50, 70, and 100 $\mathrm{V}$ respectively and using the integration mode built into the hardware to integrate the output velocity. Figure 5d shows the $50 \mathrm{~V}$ sweep, which will be used to derive the deflection versus voltage fit in the next section.

\section{RESULTS}

There is good agreement between the simulation and the experimental results (Table 1). We derived the relationship between the actual gyroscope (Figure 3a) and the levitation tester (Figure 3b). The gyroscope uses approximately eight times the number of combs for drive and sense. The gyroscope has an out of plane stiffness of $.3 \mathrm{~N} / \mathrm{m}$ whereas the tester has an out-of-plane stiffness of $1.5 \mathrm{~N} / \mathrm{m}$. A fit to the data in Figure 5d gives the relationship

$$
400 \delta_{t}^{3}-600 \delta_{t}^{2}+800 \delta_{t}=V^{2}
$$

where subscript $t$ denotes tester related parameter and $g$ denotes gyro related parameter. Eq. 7 yields only one real solution for the tester deflection $\delta_{t}$. Using $F_{t}=k_{t} \delta_{t}$ and $F_{g}=N F_{t}$, we can transform this equation into a more general equation that can be applied to a device with similar electrode geometry, but with $N$ times the number of electrodes and a different out-of-plane stiffness

$$
V^{2}=a_{1}\left(\frac{k_{g}}{N} \delta_{g}\right)^{3}+a_{2}\left(\frac{k_{g}}{N} \delta_{g}\right)^{2}+a_{3}\left(\frac{k_{g}}{N} \delta_{g}\right)
$$

where $a_{1}=118.5, a_{2}=-266.7$, and $a_{3}=533.33$. Here, $N$ is a scale number of combs compared to the tester and $k_{g}$ is the out-of-plane stiffness of the gyroscope. For the considered gyroscope design, the static vertical deflection is 1.28 $\mu \mathrm{m}$ for a typical bias voltage of $5 \mathrm{~V}$. This is comparable to results presented in [1], where resonators of one eighth of the gyroscope size were shown to have deflections of up to 2 $\mu \mathrm{m}$ with $25 \mathrm{~V}$ bias. From Eq. 5, the resulting loss in scale factor is estimated to be $64 \%$ !

For the small dynamic oscillation about the static equilibrium, we use a transfer function of linearized parameters. The driving voltage is typically of the form $V^{2}=$ $\left(V_{D C}+V_{A C}\right)^{2}$, where the AC frequency is the in-plane natural frequency $\omega_{n}$ of the device. The only surviving in frequency band component of the electrostatic force is proportional to $2 V_{D C} V_{A C}$. Thus, the linearized transfer function can be presented as

$$
\frac{\Delta \delta(s)}{\Delta V_{A C}(s)}=\frac{\phi V_{D C}}{s^{2}+\frac{\omega_{z}}{Q} s+\omega_{z}^{2}}
$$

where $\omega_{z}$ is the out-of-plane natural frequency and $\phi$ is an unknown constant function. Linearization of Eq. 8 about 


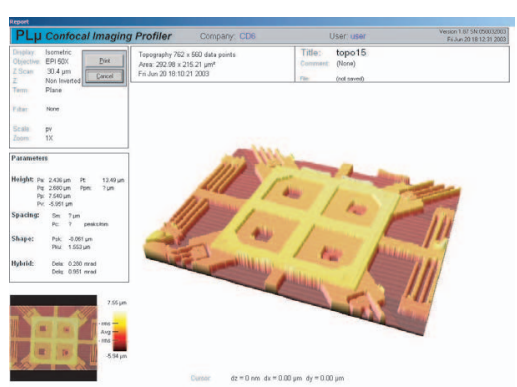

(a)

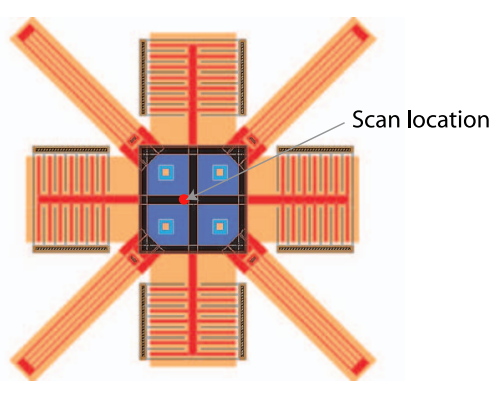

(c)

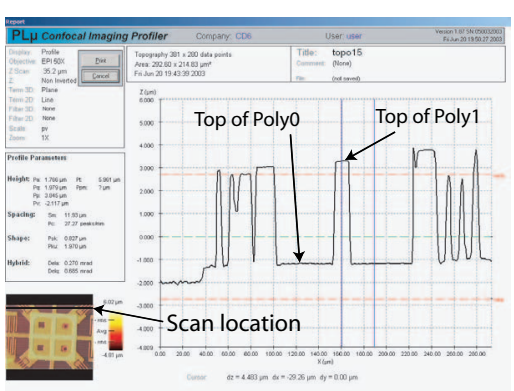

(b)

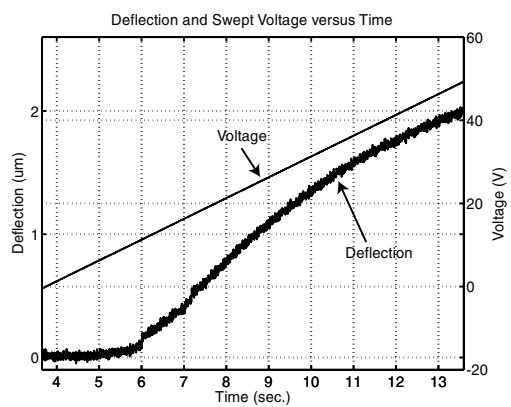

(d)

Figure 5. (a) Device is first tested using an optical profilerometer by scanning a (b) cross section of of the device's beams under varying voltages. (c) The second experiment is under a vibrometer which scans the device at a single point. (d) The device is swept under a low frequency voltage signal, giving the deflection versus voltage profile.

Table 1. Comparison of deflection values

\begin{tabular}{|c|c|c|c|}
\hline Voltage & Simulation & Confocal Mic. & Vibrometer \\
\hline $50 \mathrm{~V}$ & $1.7 \mu \mathrm{m}$ & $1.6 \mu \mathrm{m}$ & $2.0 \mu \mathrm{m}$ \\
\hline $70 \mathrm{~V}$ & $2.2 \mu \mathrm{m}$ & $2.0 \mu \mathrm{m}$ & $2.4 \mu \mathrm{m}$ \\
\hline $100 \mathrm{~V}$ & $2.7 \mu \mathrm{m}$ & $2.3 \mu \mathrm{m}$ & $2.7 \mu \mathrm{m}$ \\
\hline
\end{tabular}

the equilibrium deflection and voltage results in $\Delta \delta=.52$ $\Delta V_{A C}$. Substituting this into Eq. 9 with $\mathrm{s}=0$, corresponding to a low frequency response, gives the value for $\phi$. The gyroscope has an out-of-plane frequency of $\omega_{z}=4000 \mathrm{~Hz}$, an in-plane drive frequency of $\omega_{n}=20000 \mathrm{~Hz}$, out-of-plane $Q$ factor in vacuum of 1000 , and uses an AC drive voltage of amplitude $.25 \mathrm{~V}$. Solving for oscillation magnitude gives a value of $.005 \mu \mathrm{m}$, a negligibly small amount in this case. Since this gyroscope operates with nonlinear parallel plates and is limited to a drive oscillation amplitude of $.67 \mu \mathrm{m}$, the cross axis sensitivity is $.7 \%$ of the main axis sensitivity. This small value of cross axis sensitivity is due to the large frequency split between operational and out-of-plane frequencies. For example, for a device with a $15000 \mathrm{~Hz}$ outof-plane frequency, the cross axis sensitivity would increase to $25 \%$.

\section{CONCLUSIONS}

We have derived a model which relates the effects of levitation forces to scale factor variation and cross axis sensitivity. Through simulation and experimental verification of a levita- tion tester, it was shown that levitation forces can have a significant effect on the performance of surface micromachined gyroscopes. The results of the simulation and experiments were applied to a typical example of a surface micromachined gyroscope which show levitation forces causing a $64 \%$ change in scale factor and introduce a $.7 \%$ cross axis sensitivity. Compensation strategies [1] are necessary to reduce these effects in order to achieve high grade inertial sensors.

\section{ACKNOWLEDGEMENTS}

This work is supported in part by the National Science Foundation Grant CMS-0409923, program managers Dr. ShihChi Liu and Dr. Masayoshi Tomizuka.

\section{REFERENCES}

[1] W. C. Tang, M. G. Lim, and R. T. Howe, "Electrostatic comb drive levitation and control method," Journal of Microelectromechanical Systems, vol. 1, no. 4, pp. 170-178, December 1992.

[2] W. Geiger, B. Folkmer, U. Sobe, H. Sandmaier, and W. Lang, "New designs of micromachined vibrating rate gyroscopes with decoupled oscillation modes," Sensors and Actuators A, vol. 66, pp. 118-124, April 1998.

[3] A. Shkel and R. T. Howe, "Micro-machined angle-measuring gyroscope," November 2002, U.S. Patent 6,481,285.

[4] http://www.sensofar.com.

[5] http://www.polytecpi.com. 